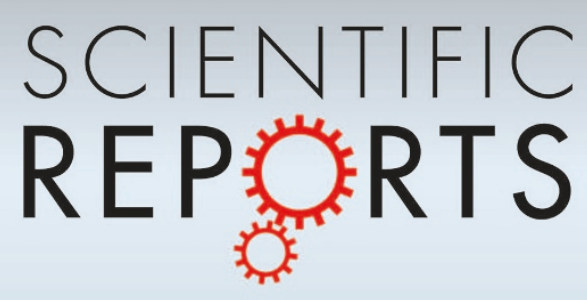

OPEN

SUBJECT AREAS:

ELECTRICAL AND

ELECTRONIC

ENGINEERING

OPTOELECTRONIC DEVICES AND COMPONENTS

Received

21 January 2014

Accepted

7 April 2014

Published

9 May 2014

Correspondence and requests for materials should be addressed to

D.A.G. (dgenov@ LaTech.edu)

\title{
Terahertz Optoelectronics with Surface Plasmon Polariton Diode
}

\author{
Raj K. Vinnakota \& Dentcho A. Genov \\ College of Engineering and Science, Louisiana Tech University.
}

The field of plasmonics has experience a renaissance in recent years by providing a large variety of new physical effects and applications. Surface plasmon polaritons, i.e. the collective electron oscillations at the interface of a metal/semiconductor and a dielectric, may bridge the gap between electronic and photonic devices, provided a fast switching mechanism is identified. Here, we demonstrate a surface plasmon-polariton diode (SPPD) an optoelectronic switch that can operate at exceedingly large signal modulation rates. The SPPD uses heavily doped $p$ - $n$ junction where surface plasmon polaritons propagate at the interface between $n$ and $p$-type GaAs and can be switched by an external voltage. The devices can operate at transmission modulation higher than $98 \%$ and depending on the doping and applied voltage can achieve switching rates of up to $1 \mathrm{THz}$. The proposed switch is compatible with the current semiconductor fabrication techniques and could lead to nanoscale semiconductor-based optoelectronics.

T

he demands for faster data transfer and processing has increased dramatically over the past five decades. This need led to enormous advances in the semiconductor industry and a continuous progression towards smaller, faster, and more efficient electronic devices ${ }^{1}$. The scaling of the electronic devices also brought about a myriad of challenges. The most daunting problems inhibiting significant increases in the processor speed are thermal and resistor-capacitor (RC) delay time, associated with the electronic interconnections and scaling of the devices ${ }^{2}$. Over the past few years saturation in the microprocessor clock speed has been observed due to the scaling issues and excessive heat dissipation ${ }^{1}$.

On the other hand, optical interconnects possess an unimaginable data carrying capacity, which may allow photonic components to mitigate the present bottlenecking in increasing the computational speed ${ }^{2}$. However, their implementation is hindered by the physical size or dimensional mismatch between electronic and dielectric photonic components. Moreover it is expected that CMOS foundries will further decrease the feature sizes on silicon chips, ultimately down to $10 \mathrm{~nm}$. Such reduction in size would further increase the dimensional mismatch between the electronic and optical elements, as the size of the dielectric photonic devices is restricted by the diffraction limit ${ }^{2}$. Moreover, miniaturization introduces several problems including dielectric break down, hot carriers and short channel effects, that degrade device reliability ${ }^{3}$.

The Surface Plasmon Polaritons (SPPs), spatially confined transverse magnetic (TM) electromagnetic modes propagating at the metal-dielectric interfaces, offer the bandwidths of photonic devices and physical dimensions shared with nanoscale electronics ${ }^{2,4-7}$. The potential of plasmonics to bridge the gap between electronics and photonics is now well recognized by the scientific community with a large number of investigators working in the field of plasmonics ${ }^{8}$. In 2008 Brongersma et al. demonstrated all-optical switch based on SPP waveguide that uses metallic (passive) nanostructures coupled with active PMMA films with photochromic molecules?. Unfortunately, the switching rates of the photochromic molecules were low $(\sim 20 \mathrm{~ns})^{9}$. The same group also proposed extremely compact gain-assisted plasmonic switch consisting of a gold-air-gold plasmonic waveguide side-coupled to a cavity filled with a semiconductor ( $\mathrm{InGaAsP}$ ) gain material ${ }^{10}$. A metal-oxide-Si field effect plasmonic modulators and all-optical modulation by plasmonic excitation of CdSe quantum dots have been investigated showing moderate transmission modulation at visible and telecommunication frequencies ${ }^{11,12}$. Recently, a THz all-optical switch based on a carbon nanotube metamaterial has been proposed, however the device shows a rather low transmission modulation of less than $10 \%{ }^{13}$.

In this article we propose a new optoelectronic device which we refer to as a Surface Plasmon Polariton Diode (SPPD). The SPPD demonstrates active control of SPPs at the interface between doped $n$-type and $p$-type semiconductors. While the propagation of SPPs on metal surfaces is well understood, not much attention has been paid on the fact that due to the extremely high plasma frequencies inherent to noble metals (for silver $\omega_{p}=$ $9 \mathrm{eV}$ ) there is a substantial bandwidths mismatch with electronics that cannot be easily bridged. Here we show that use of highly doped semiconductor material (GaAs) can serve three distinct purposes: $(i)$ act as a metal-like 


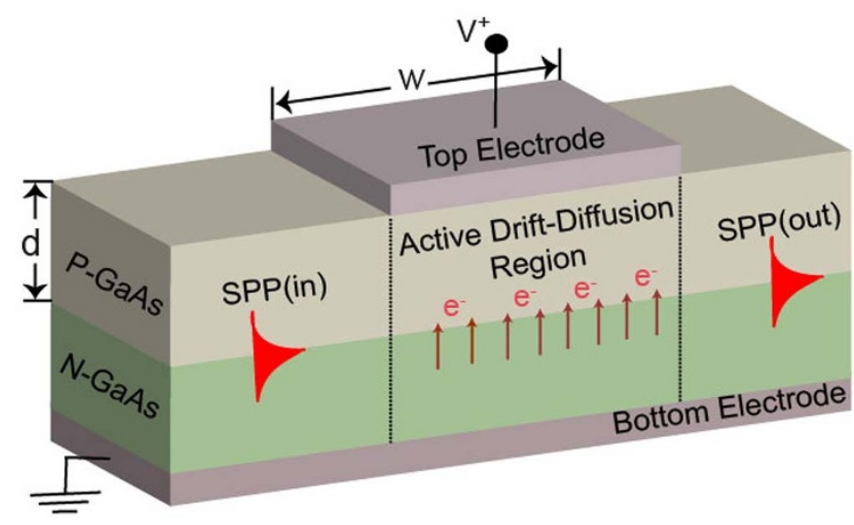

Figure $1 \mid$ Basic schematic of a Surface Plasmon Polarition Diode (SPPD).

interface allowing SPP propagation, (ii) provide faster electronic SPP switching facilitated by a $p-n$ junction, and (iii) provides tunable $\mathrm{THz}$ operational frequency range. More importantly, due to strong localization of the SPP at the $p-n$ junction, the SPPD can exhibit extremely fast (up to $1 \mathrm{THz}$ ) switching rates, has relatively small size and can be used to develop the fundamental logic for prospective use in optoelectronic data processing.

Figure 1 shows a basic schematic of the proposed SPPD constructed of a highly doped $p-n$ junction formed between two control electrodes. The SPPD basic principle of operation follows. For frequencies below the plasma frequency, $\omega_{p}$, the semiconductors behave as metals and can be tuned by doping or by applying an external bias. The metal-like properties of the semiconductors allow propagation and active control of SPPs at the $p$ - $n$ junction. Under zero-bias the SPPs propagate freely at the metallurgic junction and across the drift-diffusion zone, establishing the ON-state of the devices. With the introduction of the external bias electrons are injected in the $p$-type region, dramatically altering its optical properties from dielectric-like to metal-like. This prohibits SPP propagation across the active zone and establishes the OFF-state of the device. The SPPD can be designed using a large variety of semiconductor materials. Here we use GaAs instead of Si due to its low effective electron mass, higher plasma frequency, and high mobility.

Figure 2(a) depicts the SPPs dispersion curves $\omega=\omega\left(k_{s p p}\right)$ (where $k_{s p p}$ is the SPP wavevector) for two doping concentrations (see Methods). At low frequencies the SPP wave vector coincides with that in air while at frequencies close to the surface plasmon frequency $\omega_{s p}=\omega_{p} / \sqrt{2 \varepsilon_{b}}\left(\varepsilon_{b}=12.9\right.$ is the bound electrons contribution to the $p$-type layer permittivity ${ }^{16}$ ) it experiences a "resonant" type of behavior with $k_{S P P} \gg 2 \pi / \lambda$, where $\lambda$ is the wavelength in free space. Consequently, the effective mode wavelength is dramatically reduced below the diffraction limit in the semiconductor, allowing for the SPPD to be substantially smaller compared to similar devices based on bulk optical modes. We must note that when choosing the SPPD operation frequency it is important to consider the tradeoff between device size and mode dissipation. In this work we fix the operation frequency at $35 \mathrm{THz}$ which allows for a substantial decrease of the mode size less than $300 \mathrm{~nm}$ in a direction perpendicular to the $p-n$ junction, without imparting extensive propagation loses. Furthermore, at low doping concentrations the depletion region can act as a gap waveguide for weakly bounded bulk modes ${ }^{14}$. For the doping levels considered in this work $\left(N_{D}>10^{19} \mathrm{~cm}^{-3}\right)$ the depletion region is only a few nanometers in size ${ }^{15}$, and the excitation of such weakly bound photonic modes is strongly inhibited (see Fig. 2(a)).

The SPPD operation characteristics are first studied under steady state conditions. We use a self-consistent numerical model that a)

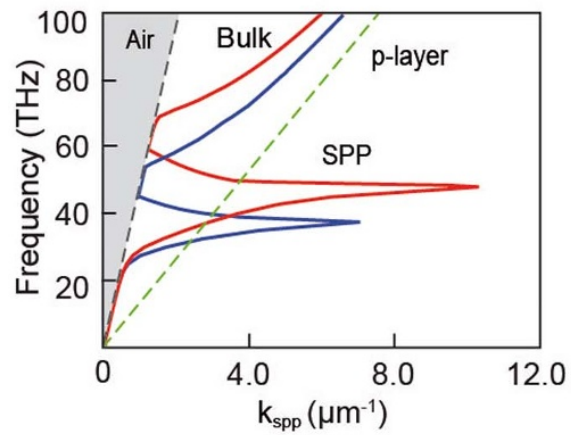

c)

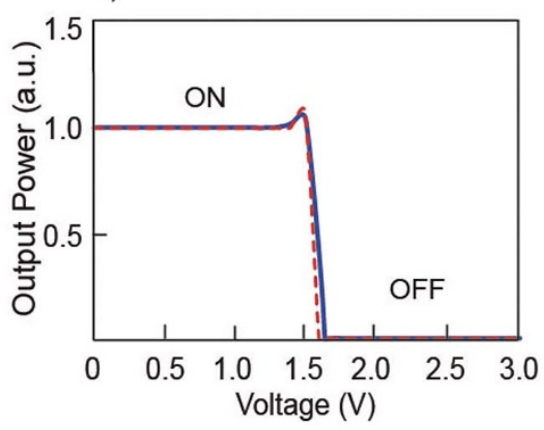

b)

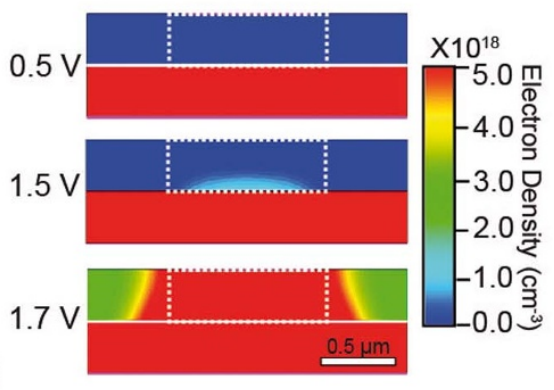

d)

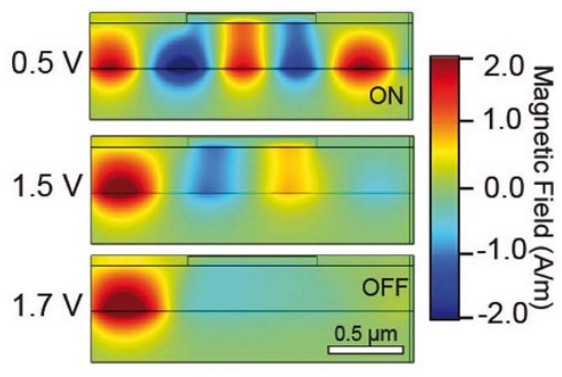

Figure 2 SPPD steady-state response. (a), The SPPD dispersion curves in air/p-type/ $n$-type GaAs waveguide configuration for two doping

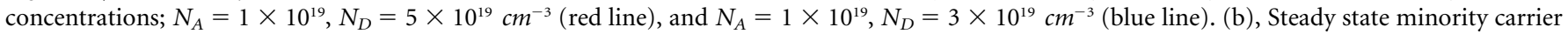

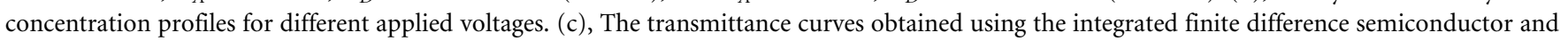

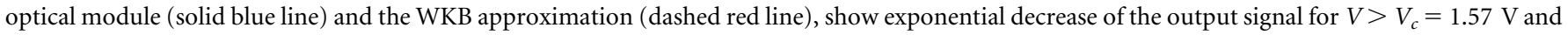
(d), SPP propagation across the SPPD drift-diffusion zone obtained at different applied voltages. In the calculations the operation frequency is set at $35 \mathrm{THz}$, the thickness of the $p$-type layer is $d=0.5 \mu m$ and the overall length of the active drift-diffusion region is fixed at $w=2 \mu m$. 
a)

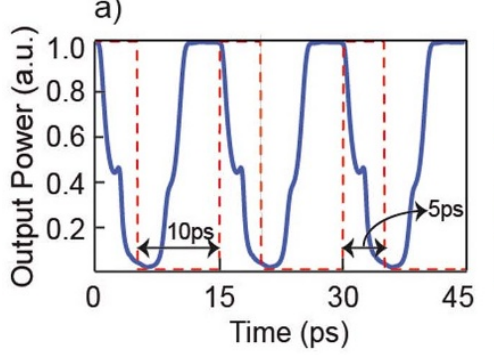

b)

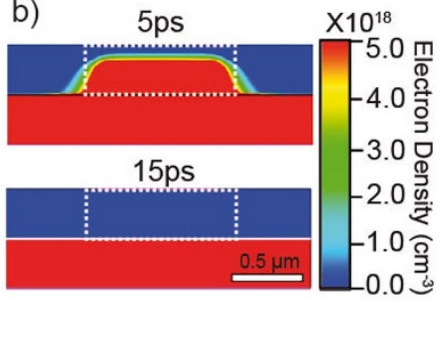

c)

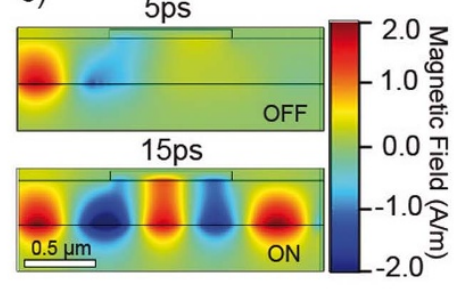

Figure 3 SPPD switching. (a), SPPD transmittance curve (solid blue line) under step-type of input voltage (dashed red line). The signal is repetitively switched following the external voltage. (b), Electron concentration and (c), Magnetic field across the device at two different times corresponding to the OFF and ON states of the device. In the calculations the operation frequency is set at $35 \mathrm{THz}$, and the doping concentrations are $N_{A}=N_{D}=5 \times$ $10^{19} \mathrm{~cm}^{-3}$.

merges the electronic response, obtained from finite difference (FD) integrated circuits semiconductor code (SENTAURUS, Synopsys Inc.), and the optical response of the device simulated with a commercial electromagnetic code (COMSOL Multiphysics). Under zero applied bias the electron concentration in the $p$-type layer is low $n \approx n_{0}=n_{i}^{2} / N_{A}$, where $n_{i}=2.25 \times 10^{6} \mathrm{~cm}^{-3}$ and $N_{A}$ is the acceptor concentration, causing this layer to behave as a dielectric (See Fig. 2(b) $)^{16}$. Concurrently, the uninterrupted SPP propagation establish the "ON" state of the device (See Fig. 2(c,d)). If forward bias is applied the electrons drift from the $n$-type layer into the $p$-type layer, and exponentially increase the minority concentration in close proximity to the metallurgic junction $n \approx n_{0} e^{V / V_{T}}$, where $V_{T}=k_{B} T / q=$ $0.026 \mathrm{~V}$ is the thermal voltage). In the presence of sufficient bias $\left(V>V_{c}\right)$ the electron concentration in the drift-diffusion zone can surpass a critical value at which point the permittivity of the $p$-doped layer becomes negative $\left(\varepsilon_{p}(\omega)<0\right)$, and this layer becomes a metallike (the semiconductor permittivity is described by the Drude model, see Methods). The SPP could no-longer propagate through the active drift-diffusion zone, and the OFF state of the device is established (Fig. 2(c,d)). For a given operation frequency, $\omega$, the critical voltage can be obtained from the transparency condition $\omega=\omega_{p}\left(V_{c}\right) / \sqrt{\varepsilon_{b}}\left(\right.$ or $\left.\varepsilon_{p}\left(\omega, V_{c}\right)=0\right)$, and the carrier balance equation $n=n_{c} \approx n_{0} e^{V_{c} / V_{T}}$, giving

$$
V_{c}=V_{T} \ln \left[\frac{\varepsilon_{b} \omega^{2}}{\omega_{p 0}^{2}}\right]
$$

where $\omega_{p 0}=\left(n_{0} e^{2} / \varepsilon_{0} m_{e}\right)^{1 / 2}$ is the plasma frequency of the minority carriers under thermal equilibrium and zero external bias, $m_{e}$ is the electron effective mass in the $p$-type layer, and $q$ is the electron charge. For $N_{A}=5 \times 10^{19} \mathrm{~cm}^{-3}$ and $f=\omega / 2 \pi=35 \mathrm{THz}$ the critical voltage is $V_{c}=1.57 \mathrm{~V}$, which is more than an order of magnitude lower than the breakdown voltage for the device ${ }^{17}$. Due to the low effective mass of electrons in the $p$-doped GaAs $\left(m_{e}=0.067 m_{0}\right)$ the critical voltage is lower than expected for Si-based $\left(m_{e}=0.26 m_{0}\right)$ SPPDs, which leads to lower heat dissipation and further justifies the choice of using GaAs.

The SPPD switching, can be qualitatively described using the Wentzel-Kramers-Brillouin (WKB) method $^{18}$, which implemented for the SPP transmittance across the drift-diffusion region yields:

$$
T(V)=\left|e^{i \int_{0}^{w} k_{s p p}(x, V) d x}\right|^{2} \approx e^{-2 w \operatorname{Im}\left[k_{s p p}(V)\right]}
$$

where $w$ is the length of the active drift-diffusion region (roughly corresponding to the length of top electrode), and the SPP wave vector, $k_{s p p}$, which is generally position and voltage dependent due to the spatially inhomogeneous minority carrier concentration in the p-type layer. Under steady state conditions, the SPP wave vector in the drift-diffusion zone can be assumed to be spatially homogeneous and dependent only on the applied voltage $k_{s p p}(x, V) \approx k_{s p p}(V)$. As a result, the WKB approximation gives an explicit, analytical formulation of the SPP transmission across the device. For a given frequency of operation, $\omega$, and sufficiently low minority carrier concentration $k_{s p p} \in \mathbb{R}$ and $T \approx 1$. When $V>V_{c}$, the permittivity of the $p$-doped layer becomes negative $\left(\varepsilon_{p}(\omega)<0\right)$ and the SPP wave vector is complex quantity $\left(k_{s p p} \approx i\left(\frac{\omega_{p o}}{c}\right) e^{\frac{V}{2 V_{T}}} \in \mathbb{C}\right)$. Consequently the transmittance exponentially decreases with an increase in the applied voltage (see dashed line in Fig. 2c).

The prime interest in the study of the SPPD is the speed at which the optical properties of the $p$-layer switches between dielectric $\left(\varepsilon_{p}>\right.$ $0)$ to metallic $\left(\varepsilon_{p}<0\right)$ and vice-versa. The switching rate depends on the applied bias and doping concentrations and can be obtained from time-dependent calculations of the minority concentration and the corresponding SPPD propagation characteristics. Figure 3(a) shows the SPPD temporal responses under step-type input voltage where a forward bias is applied across the drift-diffusion zone for a period of 5 ps and a zero bias follows for 10 ps (see Fig. 3(a)). The SPPD transmittance curve, depicted in Figure 3(a), is obtained by FD self-consistent numerical calculations in the time domain and shows reproducible switching. Under a forward bias larger than the critical $\left(V>V_{c}\right)$, the minority carries easily overcome the potential barrier due to the space-charge region. The electron concentration rises exponentially close to the metallurgic junction. Within a few picoseconds the concentration surpasses the critical value, $n_{c}$, at which point the SPPs are no longer able to propagate (establishing the "OFF" state of the device), see Fig. 3(b)\&(c). Once the applied bias is removed the excess electrons in the $p$-layer diffuse and their concentration falls below the critical value in approximately $5 \mathrm{ps}$. At this point the SPPs can propagate through the device re-establishing the "ON" state. The results indicate that extremely fast switching can be achieved corresponding to data rates of $100 \mathrm{GHz}$ and faster. At the operational frequency of $35 \mathrm{THz}$ and a voltage of $V=4 \mathrm{~V}$ the SPPD transmission modulation is $\bmod _{d B}=10 \log _{10}\left(P_{\text {max }} / P_{\text {min }}\right)=18 \mathrm{~dB}$ (corresponding to $98.4 \%$ transmission modulation), surpassing by far what have been reported in the literature, in the case of all-optical SPP based switches ${ }^{9,11-13}$.

From Figure 2(b) and Figure 3 (b) it is evident that it is not necessary for the minority concentration to surpass the critical value within the entire drift-diffusion zone to switch the SPPD, rather, only in close proximity to the metallurgic junction. This is a fundamentally different effect compared to conventional, all-electronic diodes, where the response time is proportional to the electron fly time between the terminals. The SPPs, however, are exponentially sensitive to the dielectric environment in close proximity to the spacecharge region, the metallurgic interface, and are switched OFF within a few picoseconds once the electron concentration within 20 to $50 \mathrm{~nm}$ from the junction surpasses the critical concentration value, $n_{c}$. This fact and the almost immediate signal transfer between a set of 
SPPDs (the SPPs travel with the speed of light) is what may allow for much faster response times and negligible interconnect delay times of lumped optoelectronic circuits made of SPPD as compared to conventional diodes.

The temporal response of the device depends on the injection rate of electrons from the $n$-type into the $p$-type layer and thus on both applied voltage and doping concentrations. Figure 4(a) and 4(b) present parametric studies of the SPPD response times as function of forward bias and $p$-doping concentrations. The ON time of the device is diffusion limited and is thus independent of the initial applied bias. The OFF times, however, are governed by the drift of the electrons in the $p$-type layer and are thus inversely proportional to the applied voltage. We must caution that operation at high external biases is not encouraged since it will lead to substantial increase in power dissipation and potential device failure. Thermal analysis of the SPPD has to be performed to establish the maximum voltage that can be applied, which will be the subject of future work. Furthermore, as shown in Figure 4(b), the response times decrease exponentially with increase in the $p$-layer doping. This phenomenon is due to the dependence of $\tau_{O N}$ and $\tau_{\text {OFF }}$ on the SPP penetration depth in the $p$-type layer which varies with the doping. The effects of applied voltage and doping on the response times can be quantitatively estimated as follows.

Due to the presence of external bias, electrons drift into the $p$-layer with a velocity $v_{d} \approx \mu_{e}^{p}\left(V-V_{b i}\right) / x_{p}$, where $\mu_{e}^{p}$ is the minority carrier drift mobility in $p$-type $\mathrm{GaAs}^{19}, x_{p}$ is the thickness of the $p$-layer and $V_{b i}$ is the build-in potential ${ }^{15}$. The SPPD OFF time is inversely proportional to the drift velocity and the applied bias and can be
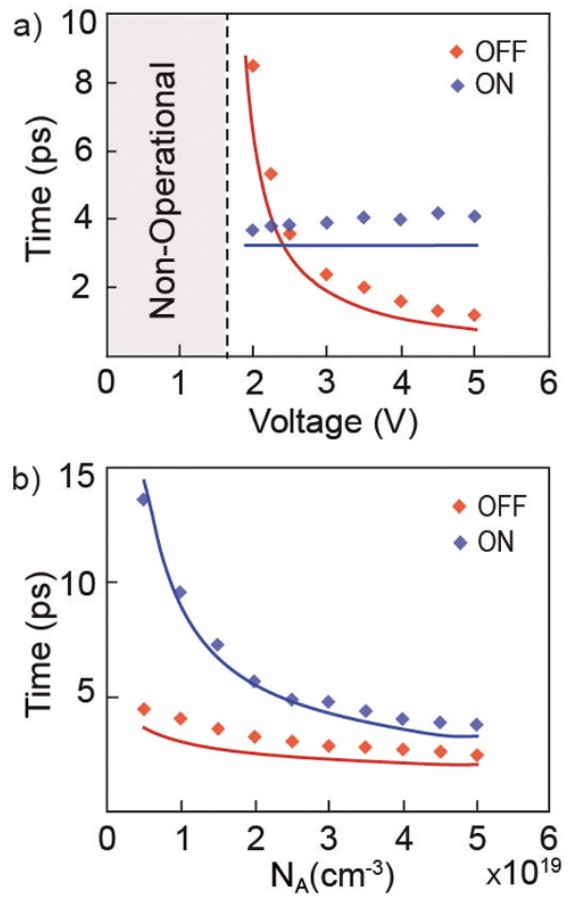

Figure 4 SPPD response times. (a), Dependence of the SPPD ON and OFF times on the applied voltage $\left(V>V_{c}\right)$. The device OFF time is dependent on the electron drift toward the electrodes and is thus inversely proportional to the applied voltage. In the calculations the SPP operation frequency is set at $35 \mathrm{THz}$, and the doping concentrations are fixed at $N_{A}=$ $N_{D}=5 \times 10^{19} \mathrm{~cm}^{-3}$. (b), The SPPD response times as function of the $p$ layer doping concentration. The doping influences both the width of the space charge region and the electron mobility thus modifies to a different extent the $\mathrm{ON}$ and $\mathrm{OFF}$ times of the device. In the figures the numerical data (diamonds) is compared to the analytical drift-diffusion model (solid lines). In the calculations the applied voltage is $V=3 \mathrm{~V}$. estimated as $\tau_{\mathrm{OFF}}=l_{s p p} / v_{d}$, where $l_{s p p}=1 / k_{z}^{s p p}=1 / \sqrt{k_{s p p}^{2}-\varepsilon_{p} k_{0}^{2}}$ is the field penetration depth of the SPP in the p-type layer (see Methods). At zero bias, the excess minority carriers in the $p$-layer are removed by the net carrier outflow from the quasi-neutral region via diffusion. Using dimensional analysis we can write the response (ON) time as $\tau_{\mathrm{ON}}=l_{\text {spp }}^{2} /\left(4 F D_{n}\right)$, where $F \approx 4$ is a shape factor and it is near-independent on the parameters under investigation, $D_{n}=(k T / q) \mu_{e}^{n}$ is the diffusion co-efficient of the electron in $p$ region, $\mu_{e}^{n}$ is the electron mobility ${ }^{20}$. The predicted response times by the above analytical considerations are shown with solid lines in Figure 4 and can be considered as the lower bounds of the actual response times. Overall, the SPPD can operate at very fast switching rates of up to $1 \mathrm{THz}$ (or 1 ps response time).

The SPPD, similarly to its all-electronic counterparts, can be potentially used to develop the fundamental unit of a microprocessor i.e., a logic gate. Due to the fast response times the SPPD may significantly improve the clock speed of an optoelectronic processor unit. In Figure 5 we propose and study possible designs of optoelectronic logic elements based on the SPPD. The logic elements are constructed by connecting three SPPs waveguides in a "Y" shape configuration in which each waveguide is controlled by a separate SPPD. The NAND gate (Figure 5(a)) is controlled by two external voltages, $V_{1}$ and $V_{2}$, applied at the input waveguides SPPDs, while the NOR gate (Figure 5(b)) is controlled by external voltages applied in series at the input and output waveguides SPPDs. In the absence of external bias $\left(V_{1}=0 \mathrm{~V}\right.$ and $\left.V_{2}=0 \mathrm{~V}\right)$ the input SPPs interact constructively at the $\mathrm{Y}$-junction, and the signal is transferred establishing the "ON" states for both NAND and NOR gates. In the presence of single external bias $\left(V_{1}=0 \mathrm{~V}, V_{2}=2 \mathrm{~V}\right.$ or $V_{1}=2 \mathrm{~V}$, $V_{2}=0 \mathrm{~V}$ ), the SPPs from one of the impute waveguides is transmitted in the case of the NAND gate ("ON" state) and reflected in the case of a NOR gate ("OFF" state). When both input SPPDs are biased, there is no transmission and both gates are in "OFF" state. The actual three dimensional magnetic field profiles calculated in correspondence to the logic tables Fig. 5 (c, f) are depicted in Fig. 5(b, e). Both electronic control (through the SPPD applied voltages) and constructive/destructive interference at the output waveguide are utilized to establish the proper input/output signal associations. One limitation for the proposed " $\mathrm{Y}$ " shaped device is it's the phase sensitivity. Specifically, the gate requires two input signal of same phase to have a constructive interference in the absence of external bias $\left(V_{1}=0 \mathrm{~V}\right.$ and $\left.V_{2}=0 \mathrm{~V}\right)$ and thus the highest power output. We must emphasize that in principle there are many possible gate configurations and the chosen " $Y$ " shape design may not be the optimal. More importantly, due to the SPPD nature of operation, the proposed logic gates are optoelectronic (not all-optic) and can be simply viewed as a fast and efficient way of controlling the propagation of surface plasmon polariton (SPP) modes at highly doped semiconductor interfaces.

In conclusion we have introduced and demonstrated a new optoelectronic switching device, i.e. surface plasmon polariton diode (SPPD), capable of operating at exceedingly high switching rates and signal modulation. The fast device response is achieved by active control of the propagation of SPPs along a highly doped $p-n$ junction. The performed steady-state and time dependent numerical analysis suggests that the SPPD can operate at signal modulation in excess of $18 \mathrm{~dB}$ and switching rates from $100 \mathrm{GHz}$ up to $1 \mathrm{THz}$. Particular designs of the fundamental logic elements ("Y" shape configuration), NAND and NOR gates, have been demonstrated.

\section{Methods}

Surface plasmon polaritions in doped semiconductors. Similar to metals, the optical response of highly doped GaAs can be characterized by the Drude model with permittivity given as $\varepsilon_{G a A s}(\omega)=\varepsilon_{b}-\omega_{p}^{2}(n) /\left(\omega^{2}+i \omega \omega_{\tau}(n)\right)$, where $\varepsilon_{b}=12.9$ is the bound electron permittivity ${ }^{16}$. An important difference to note is that both the plasma 
a)

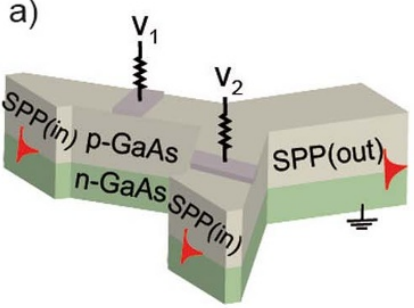

c)

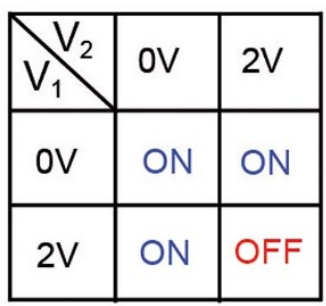

d)

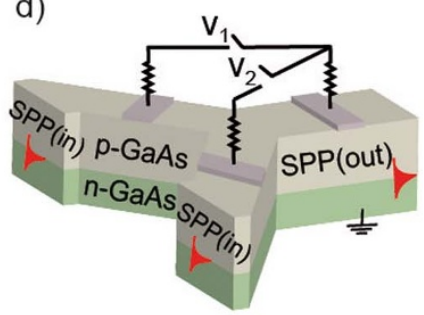

f) b)
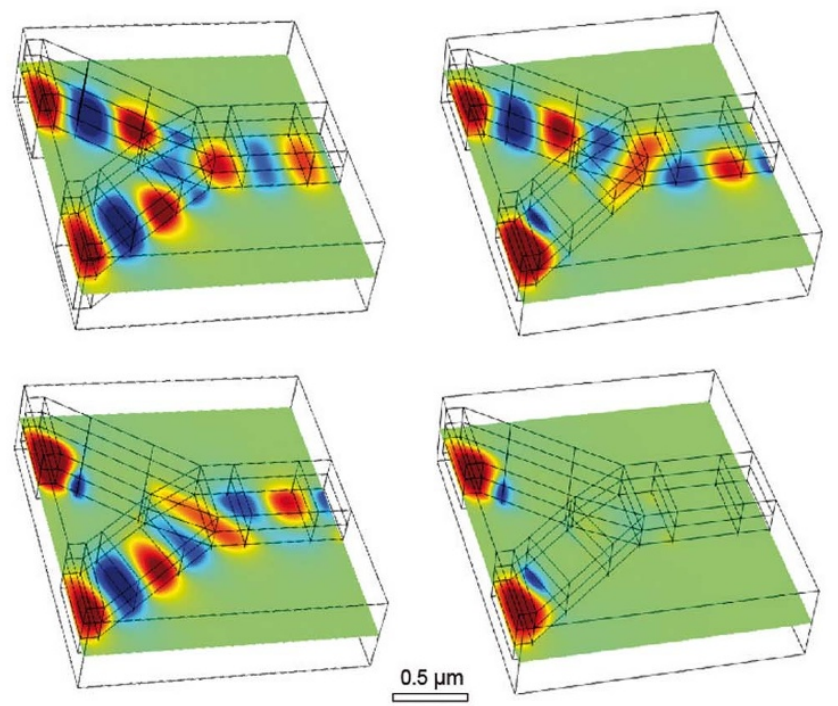

e)
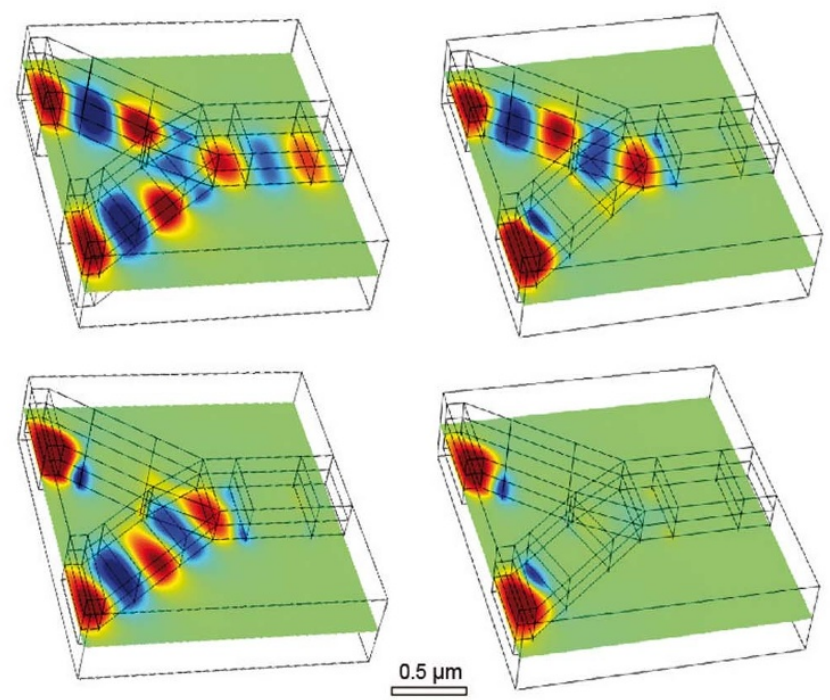

Figure 5 SPPD logic. (a), (d) Schematic of the SPPD logic (NAND and NOR) gates. (c), (f) Logic table corresponding to the NAND and NOR gates, respectively. (b), (e) SPP propagation characteristics. The control voltages $V_{1}$ and $V_{2}$ used in the calculations are specified in the logic tables.

frequency, $\omega_{p}=\left(n e^{2} / \varepsilon_{0} m_{e}\right)^{1 / 2}$, and relaxation rate, $\omega_{\tau}=e / m_{e} \mu(n)$, now depend on the doping concentration. This dependence allows for fine tuning of the optical properties of the doped GaAs for frequencies up to $200 \mathrm{THz}$ (corresponding to a maximum doping equal to $6 \times 10^{19} \mathrm{~cm}^{-321}$ ). The SPPs dispersion relation ${ }^{22}$ for a three layer planar waveguide configuration (see Fig. 1) is given as

$$
e^{-4 k_{d} d}=\frac{k_{d} / \varepsilon_{d}+k_{m} / \varepsilon_{m}}{k_{d} / \varepsilon_{d}-k_{m} / \varepsilon_{m}} \frac{k_{d} / \varepsilon_{d}+k_{a} / \varepsilon_{a}}{k_{d} / \varepsilon_{d}-k_{a} / \varepsilon_{a}}
$$

where $\varepsilon_{m}, \varepsilon_{d}, \varepsilon_{a}$ are the dielectric permittivities of $n$-layer, $p$-layer and surrounding dielectric (air), $d$ is the thickness of the $p$-layer, $k_{l}=\sqrt{k_{s p p}^{2}-\varepsilon_{l} k_{0}^{2}}, l \in\{m, d, a\}$. The SPPs wave vector $k_{s p p}$ is larger than that in free space $k_{0}=(\omega / c)$ (see Fig. 2(a)). Therefore, coupling and de-coupling of energy with the far field is inhibited due to the momentum mismatch. Consequently, the SPPs are exponentially confined to the $p-n$ junction interface ${ }^{5,6}$.

Numerical calculations. The SPPD's operation is been modeled using finite-element, steady state and time domain numerical calculations. A self-consistent model was developed by first calculating the electron and hole concentrations over the SPPD using commercial semiconductor device simulator (SENTAURUS, Synopsys Inc.). The inhomogeneous carrier's concentrations for the $n$-layer and $p$-layer (including the depletion region concentration) are then used to extract the position dependent effective permittivity of the $n$ and $p$-layer. Finite element calculations of the electromagnetic response of the diode based on COMSOL Multiphysics, follow accordingly. This process is repeated for various input parameters such as donor/ acceptor doping concentrations, applied bias, and operation frequency. A common
(MATLAB) environment is used to merge the two commercial codes, which allows automatically sharing data and performing large sets of parametric studies, critical for the optimization of the SPPD performance. The response times are estimated by fitting the transmittance data (similar to Fig. 3a) with an exponent $e^{-t / \tau}$, where $\tau$ is the switching time.

1. Sutter, H. The Concurrency Revolution. Dr Dobb's Journal (2009) at $<\mathrm{http}: / /$ www.drdobbs.com/cpp/the-concurrency-revolution/

228701465?queryText $=$ The $\% 2$ Bconcurrency\%2Brevolution $>$ (Date of access: 04/03/2014).

2. Zia, R., Schuller, J. A., Chandran, A. \& Brongersma, M. L. Plasmonics: the next chip-scale technology. Mater. Today $9,20-27$ (2006).

3. Abdeldayem, H., Frazier, D. O., Paley, M. S. \& Witherow, W. K. Recent Advances in Photonic Devices for Optical Computing. science.nasa.gov (2002) (Date of access: 04/03/2014).

4. Maier, S. a. et al. Plasmonics-A Route to Nanoscale Optical Devices (Advanced Materials, 2001, 13, 1501). Adv. Mater. 15, 562-562 (2003).

5. Genov, D. A., Oulton, R. F., Bartal, G. \& Zhang, X. Anomalous spectral scaling of light emission rates in low-dimensional metallic nanostructures. Phys. Rev. B 83, 245312 (2011).

6. Oulton, R. F., Sorger, V. J., Genov, D. A., Pile, D. F. P. \& Zhang, X. A hybrid plasmonic waveguide for subwavelength confinement and long-range propagation. Nat. Photonics 2, 496-500 (2008).

7. Ambati, M., Genov, D. A., Oulton, R. F. \& Zhang, X. Active Plasmonics: Surface Plasmon Interaction With Optical Emitters. IEEE J. Sel. Top. Quantum Electron. 14, 1395-1403 (2008). 
8. Brongersma, M. L. \& Shalaev, V. M. The Case for Plasmonics. Science 328, 440-441 (2010).

9. Pala, R. A., Shimizu, K. T., Melosh, N. A. \& Brongersma, M. L. A nonvolatile plasmonic switch employing photochromic molecules. Nano Lett. 8, 1506-1510 (2008).

10. Georgios Veronis, G. V. et al. Metal-dielectric-metal plasmonic waveguide devices for manipulating light at the nanoscale. Chin. Opt. Lett. 7, 302-308 (2009).

11. Pacifici, D., Lezec, H. \& Atwater, H. All-optical modulation by plasmonic excitation of CdSe quantum dots. Nat Photon 1, 402-406 (2007).

12. Dionne, J. A., Diest, K., Sweatlock, L. A. \& Atwater, H. A. PlasMOStor: A Metal-Oxide-Si Field Effect Plasmonic Modulator. Nano Lett. 9, 897-902 (2009).

13. Nikolaenko, A. E. et al. THz bandwidth optical switching with carbon nanotube metamaterial. Opt. Express 20, 6068-6079 (2012).

14. Bond, W. L., Cohen, B. G., Leite, R. C. C. \& Yariv, A. Observation of the dielectricwaveguide mode of light propagation in $p$ - $n$ junctions. Appl. Phys. Lett. 2, 57 (1963).

15. Sze, S. M. \& Ng, K. K. Physics of Semiconductor Devices, 74-77 (WileyInterscience, 2006)

16. Blakemore, J. S. Semiconducting and other major properties of gallium arsenide. J. Appl. Phys. 53, R123 (1982).

17. Luo, J. K., Thomas, H., Morgan, D. V., Westwood, D. \& Williams, R. H. The electrical breakdown properties of GaAs layers grown by molecular beam epitaxy at low temperature. Semicond. Sci. Technol. 9, 2199 (1994).

18. Griffiths, D. J. Introduction to quantum mechanics, 315-335 (Pearson Prentice Hall, 2005).

19. Tiwari, S. \& Wright, S. L. Material properties of p-type GaAs at large dopings. Appl. Phys. Lett. 56, 563-565 (1990).

20. Rode, D., Willardson, R. K. \& Beer, A. C. Semiconductors and Semimetals. 10, 1 (Academic Press, 1975).
21. Schubert, E. F., Gilmer, G. H., Kopf, R. F. \& Luftman, H. S. Maximum concentration of impurities in semiconductors. Phys. Rev. B 46, 15078-15084 (1992).

22. Maier, S. A. Plasmonics: Fundamentals and Applications, 30-32 (Springer, Oct 29 2010).

\section{Acknowledgments}

We would like to thanks Dr.A.Khaliq, Venkatesh PappaKrishnan and Brandon Touchet for useful discussions and inputs. This work has been supported by the Louisiana Board of Regents under contract \#LEQSF (2011- 41214)-RD-A-18.

\section{Author contributions}

D.A.G. conceived the idea and supervised this study. R.K.V. performed the simulations and calculations. All authors contributed in developing the theory and writing the manuscript.

\section{Additional information}

Competing financial interests: The authors declare no competing financial interests.

How to cite this article: Vinnakota, R.K. \& Genov, D.A. Terahertz Optoelectronics with Surface Plasmon Polariton Diode. Sci. Rep. 4, 4899; DOI:10.1038/srep04899 (2014).

This work is licensed under a Creative Commons Attribution-NonCommercialNoDerivs 3.0 Unported License. The images in this article are included in the article's Creative Commons license, unless indicated otherwise in the image credit; if the image is not included under the Creative Commons license, users will need to obtain permission from the license holder in order to reproduce the image. To view a copy of this license, visit http://creativecommons.org/licenses/by-nc-nd/3.0/ 